Supporting Information

\title{
A Freestanding Laser-Assisted Reduced Graphene Oxide Micro-Ribbon Textile Electrode Fabricated on Liquid Surface for Supercapacitors and Breath Sensors
}

HaoTian H. Shi ${ }^{\dagger}$, Sumyung Jang ${ }^{\dagger}$, and Hani. E. Naguib ${ }^{\dagger}, \ddagger, * *$

${ }^{\dagger}$ Department of Mechanical Engineering, University of Toronto, 5 King's College Road, Toronto, Ontario, M5S 3G8, Canada;

* Department of Materials Science \& Engineering, 27 King's College Circle, Toronto, Ontario, M5S 1A1, Canada; Institute of Biomaterials and Biomedical Engineering, University of Toronto, 164 College Street, Toronto, Ontario, M5S 3G9, Canada;

${ }^{*}$ Corresponding author. Email Address: naguib@mie.utoronto.ca

KEYWORDS: Reduced Graphene Oxide Micro-Ribbons; Laser Reduction; Liquid Surface; Textile Supercapacitors; Breathing Sensors 


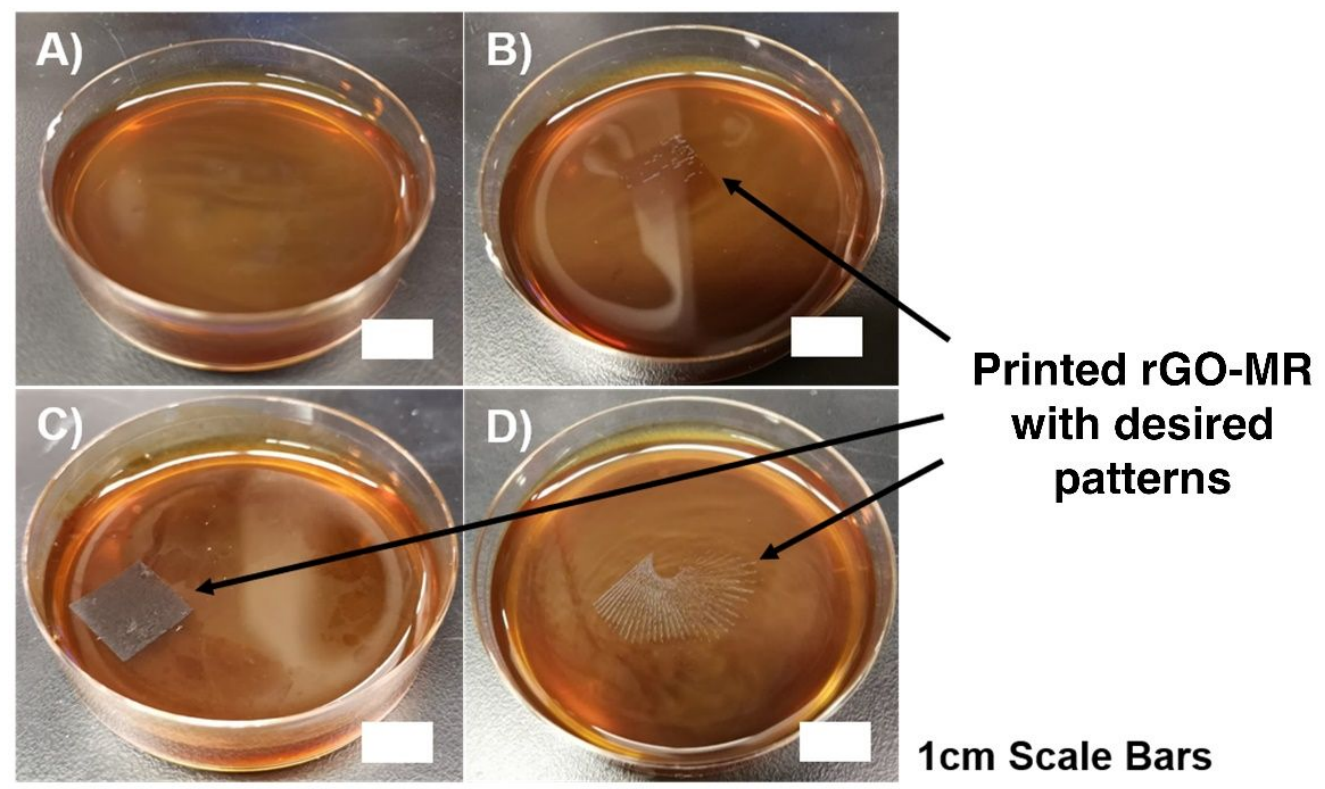

Figure S1: A variety of patterns printed by different laser parameters and pre-determined patterns A) The GO solution with only formations of GO-LC structures on the liquid surface; B) The fine lines of a rectangular pattern printed with low laser power (i.e. $<10 \%$ of $200 \mathrm{~mW}$ rated power); C) A coherent rectangular pattern printed with $15 \%$ of $200 \mathrm{~mW}$ rated power; D) Various shapes and patterns can be printed as desired.

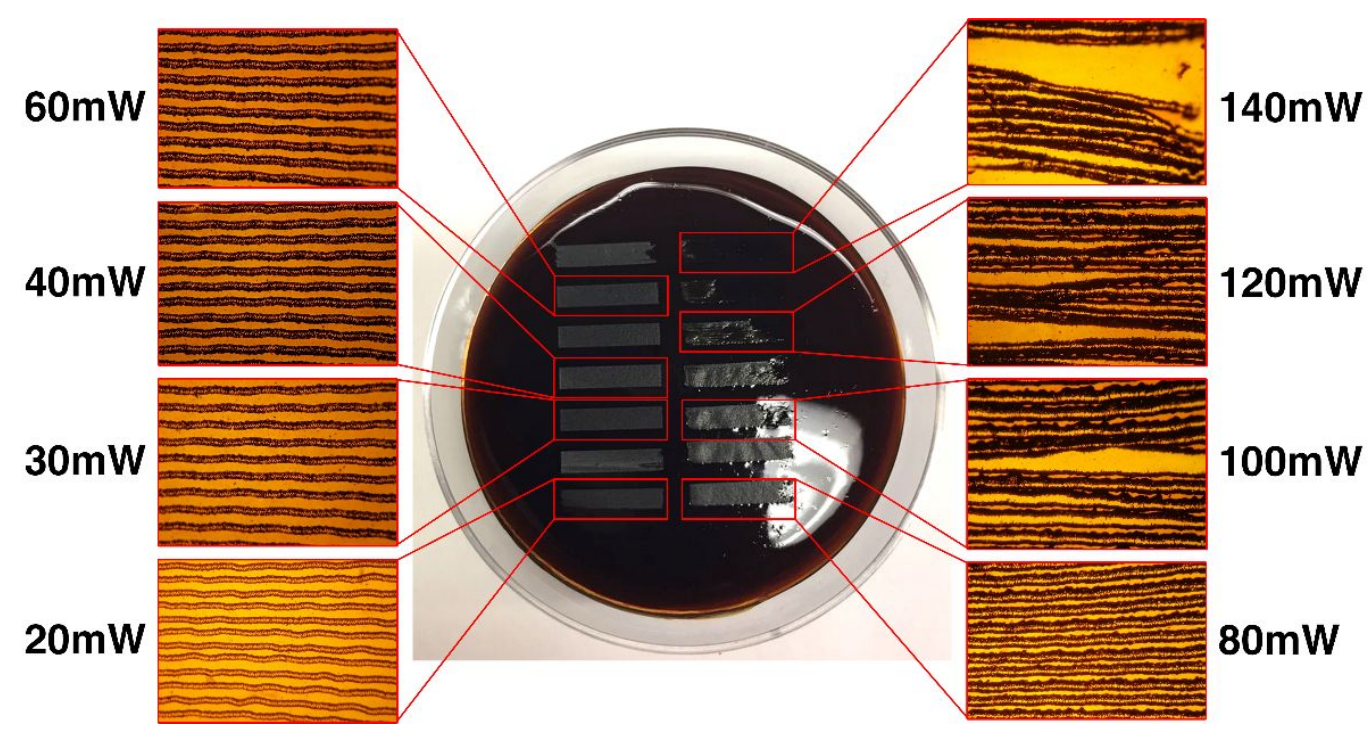

Figure S2: A comparison with a range of laser powers in creating the rGO-MR samples. Low laser powers created thin rGO-MR lines, however, lacks structural integrity due to insufficient reduction. At higher laser powers, the heat from the laser focal point burns the sample and therefore disrupting the rGO-MR network. 

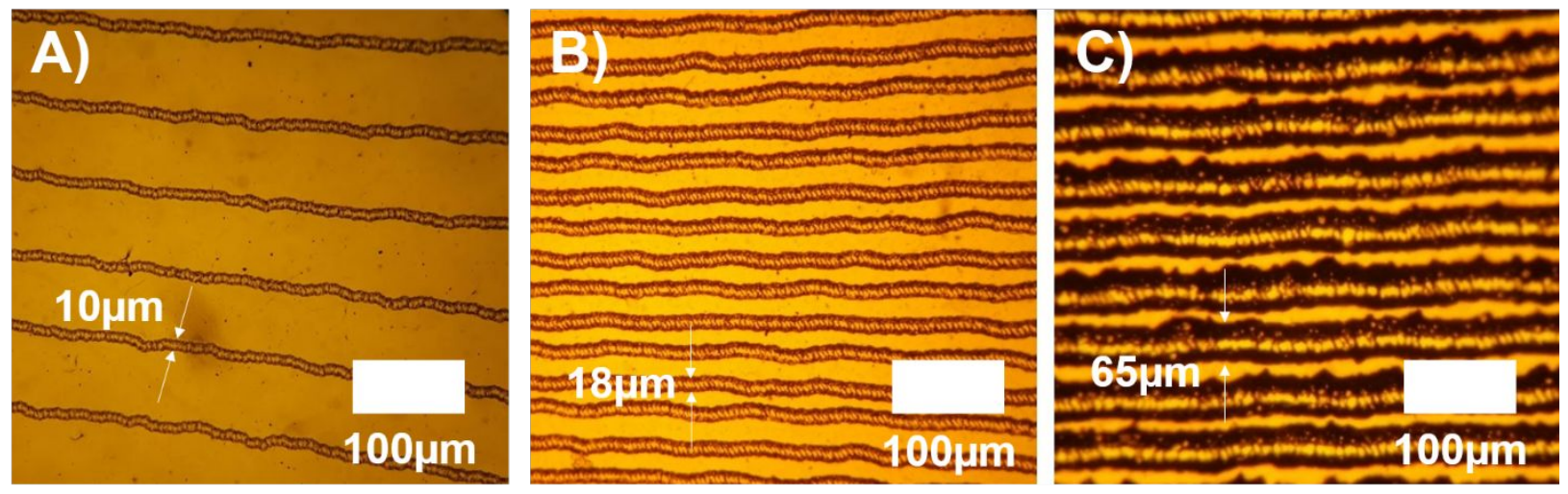

Figure S3: The diameters of the rGO-MR can be tuned by changing the laser focus, laser power, and the scan speeds. The diameters here are controlled solely based on the laser power since higher power leads to larger spread of photochemical reduction and the various diameter outcomes are demonstrated: A) $10 \mu \mathrm{m}$; B) $18 \mu \mathrm{m}$; and C) $65 \mu \mathrm{m}$.
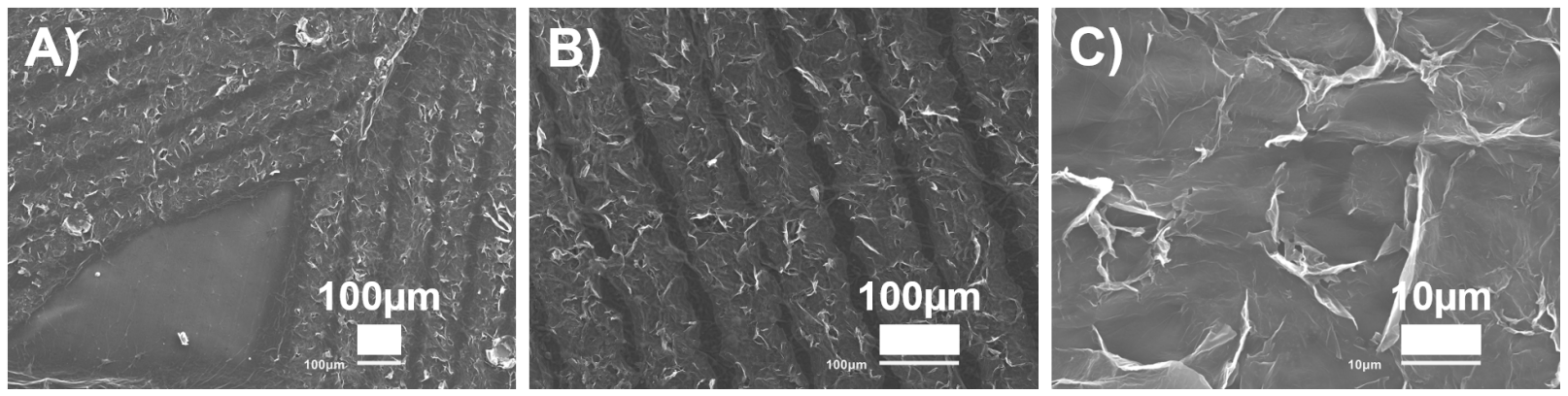

Figure S4: Further SEM studies of printed rGO-MR patterns: A) By folding the film in the liquid upon itself, an arrow shape was created; B) \& C) Additional zoomed SEM images of the $r G O-M R$ structures showing graphene-like morphology.
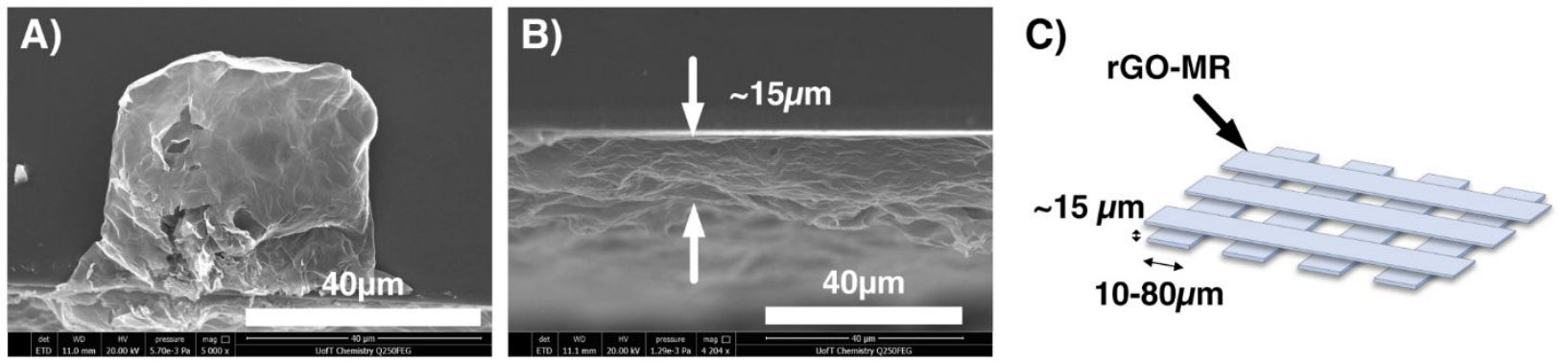

Figure S5: Thickness determination of the rGO-MR samples A) A single rGO-MR fiber that has been protruded out of the substrate, showing the an edge of the bent rGO-MR with a diameter of around $45 \mu \mathrm{m}$; B) The rGO-MR textile has been attached on a glass substrate and was positioned at $90^{\circ}$ angle to the horizontal, and by taking consecutive SEM scans, it was found that the average thickness of the rGO-MR fibers is around $15 \mu \mathrm{m}$; C) The schematics showing the rGO-MR networks in the case of a crisscross structure with dimensions indicated. 

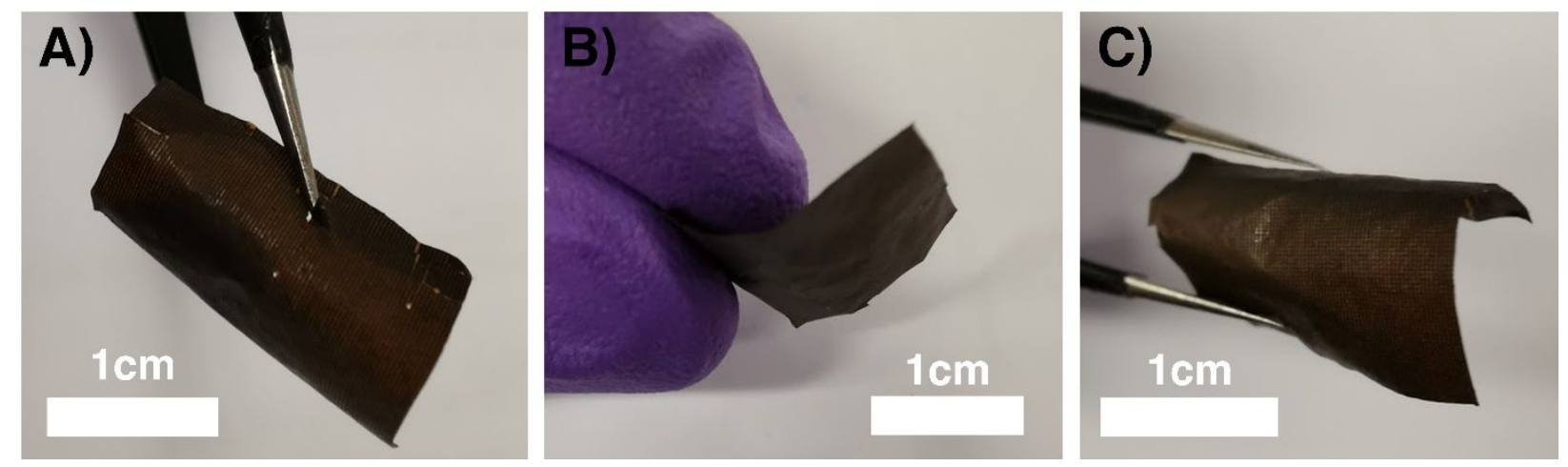

Figure S6: The single crisscross scanned rGO-MR electrodes demonstrate an exceptionally thin formfactor with a good flexibility and bendability. It was shown that it can withstand gentle bending cycles without permanently deforming.

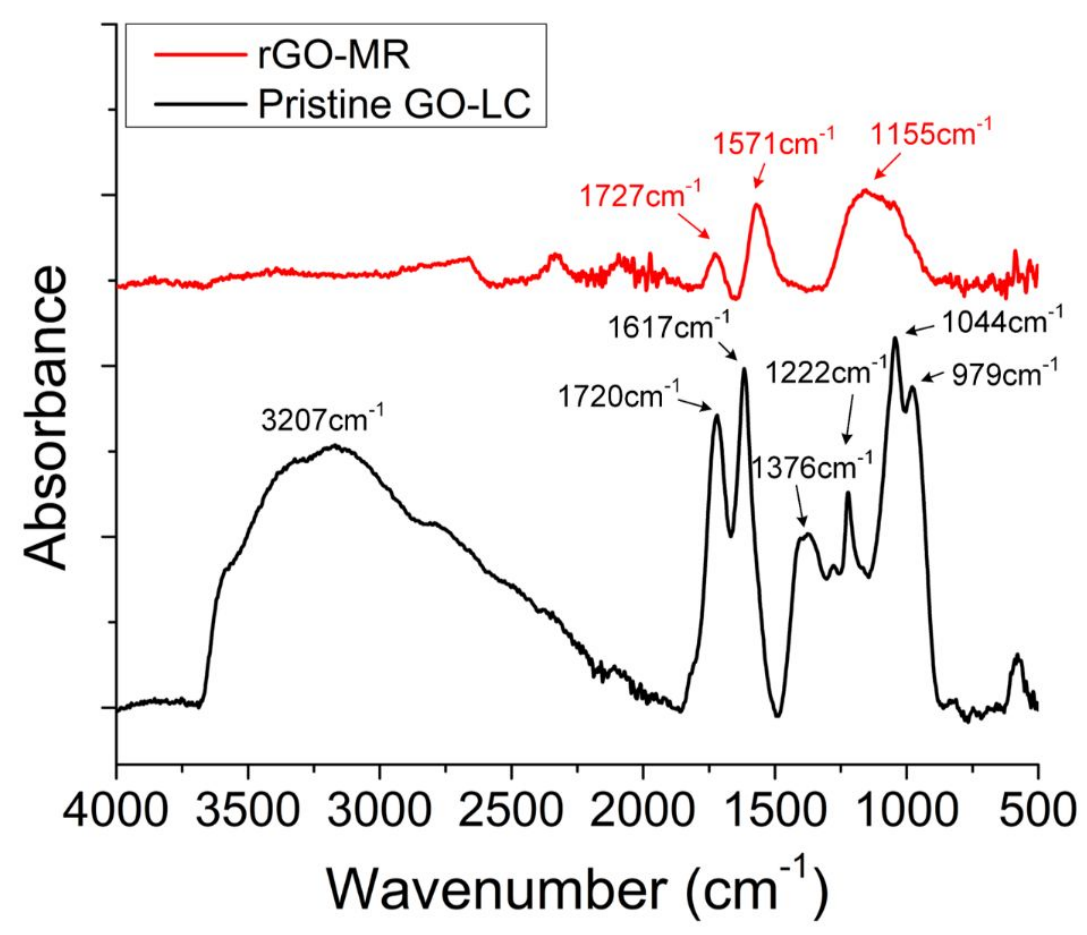

Figure S7: Fourier Transform Infrared Spectroscopy (FTIR) spectra of the rGO-MR samples in comparison with the pristine GO-LC films: A direct comparison between the $r G O-M R$ vs. the pristine GO-LC samples demonstrating the optimized reduction process taking place; 

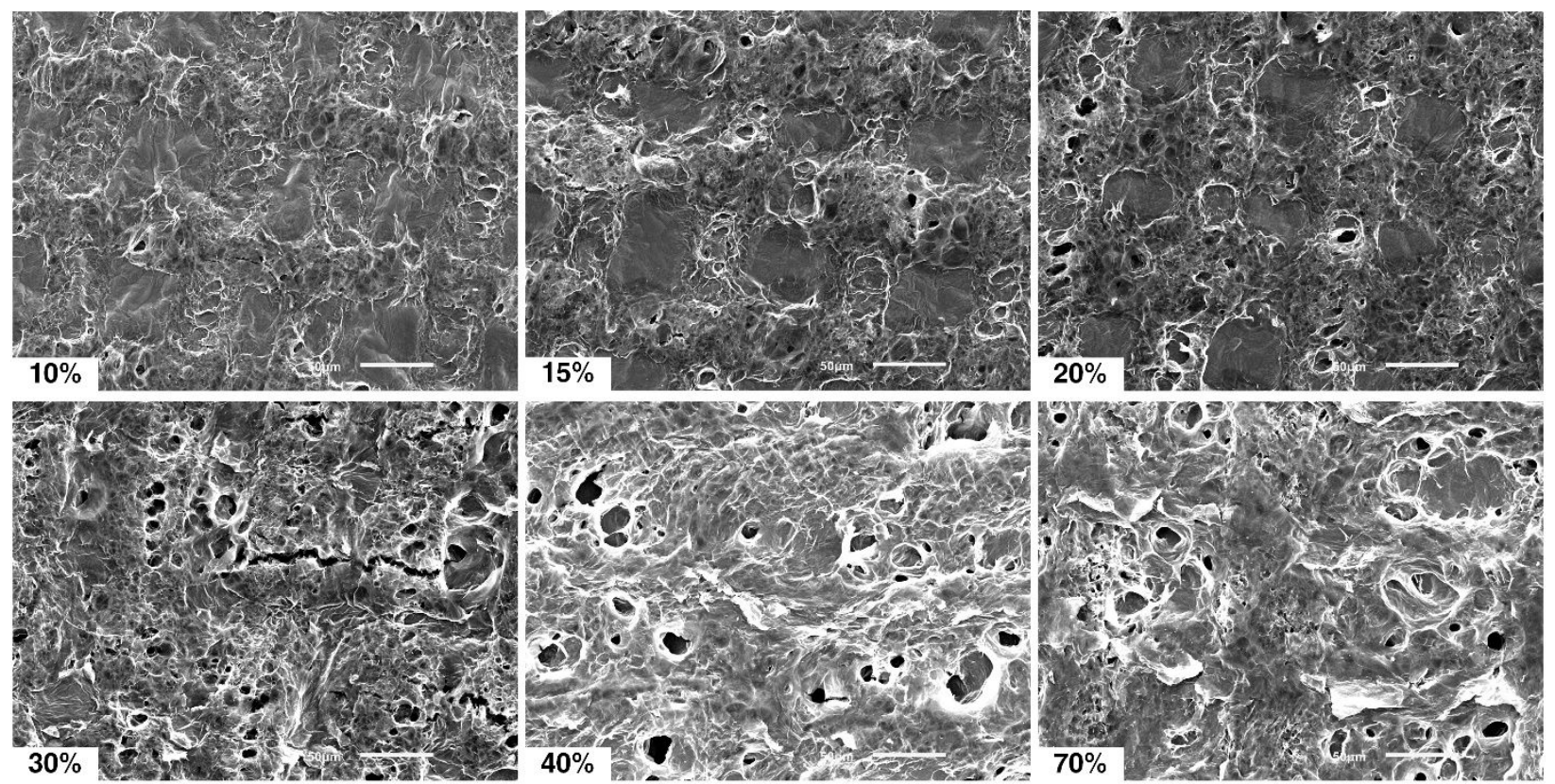

Figure S8: SEM images of $r G O-M R$ films created with a range of laser powers from 10\% to $70 \%$ $(20 \mathrm{~mW}$ to $140 \mathrm{~mW})$ showing the structural integrity was deteriorated as the laser power increased beyond 20\% $(40 \mathrm{~mW})$, which resulted in burning and creation of cracks and other discontinuities.

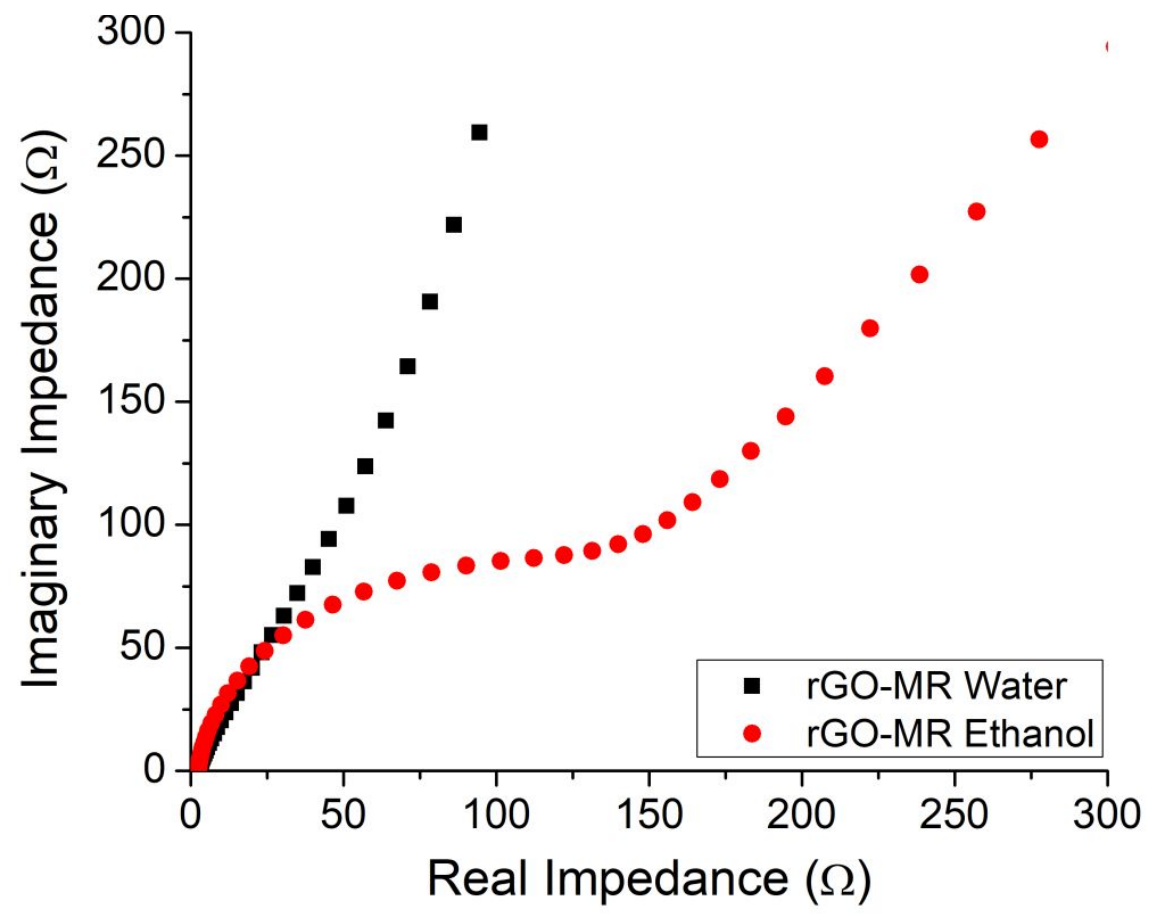

Figure S9: The Nyquist plot of the electrochemical impedance spectroscopy (EIS) comparing the rGO-MR textile electrode washed with distilled water and ethanol. 